\title{
Cat States and Single Runs for the Damped Harmonic Oscillator
}

\author{
Peter Goetsch, Robert Graham, and Fritz Haake \\ Fachbereich Physik, Universität-Gesamthochschule Essen, 45117 Essen, Germany
}

\begin{abstract}
We discuss the fate of initial states of the cat type for the damped harmonic oscillator, mostly employing a linear version of the stochastic Schrödinger equation. We also comment on how such cat states might be prepared and on the relation of single realizations of the noise to single runs of experiments.
\end{abstract}

Typeset using REVTEX 


\section{INTRODUCTION}

The conventional quantum description of open systems employs density matrices and their nonunitary evolution equations, i. e. master equations. An equivalent description can be given in terms of wave functions, at the expense of introducing damping and noise terms in the Schrödinger equation (see e. g. [1 22]). For a given open system, both the master equation and the corresponding stochastic Schrödinger equation can be derived by treating the interaction, usually weak, with an environment.

Whether one deals with a master equation or the equivalent stochastic Schrödinger equation is mostly a matter of convenience. Less work is involved in determining $N$ components of a state vector for a single realisation of the noise process rather than $N^{2}$ elements of a density matrix; however, many realisations of the noise must be followed in order to establish the full information stored in the density matrix.

Apart from occasional computational convenience the stochastic Schrödinger equation has a certain conceptional appeal. Recent experiments on individual ions or atoms stored in electromagnetic traps have revived the old question about what quantum mechanics has to say about the course of a single run of an experiment. The so-called quantum jumps of an atom into or out of a metastable state and the accompanying random-telegraph modulation of a fluorescence signal, for instance, strengthen our interest in a formulation of dissipative quantum dynamics where random jumps are naturally reflected in the time dependence of the wave function.

The stochastic Schrödinger equation is particularly welcome to some researchers interested in quatum measurement theory. The seemingly scandalous effective collapse of a superposition to a mixture upon measurement is by now rather well understood, even on the basis of exactly solvable models [23 25]. However, it is still 
esthetically satisfying to see the wavefunction of a pointer variable evolve, in each realization of the noise, to one particular distinguished displacement corresponding to one eigenvalue of the measured observable of some microsystem; and to see many independent realisations of the noise build up the relative frequencies of the possible outcomes.

Other peculiarities of quantum mechanics, like Schrödinger's cat and the EPR paradox, appear under an interesting new perspective in the description by a stochastic Schrödinger equation. The fate of Schrödinger's cat was recently investigated numerically in a simple model in this manner [26]. Previous treatments of such problems using the density matrix [27,28] were thus complemented in rather more intuitive ways.

We here take up the cat problem again. We present a rigorous solution of a stochastic Schrödinger equation for the damped harmonic oscillator, starting from an initial state of the cat type. It is most gratifying to see how the wavevector chooses randomly between the two possible and "macroscopically" distinguishable locations. The choice is made on the so-called decoherence time scale $t_{\mathrm{dec}}$ which is shorter than the mechanical damping time by a factor measuring the difference between the two initial locations. For each realization of the noise, jumps between the two locations become exceedingly unlikely after several units of $t_{\mathrm{dec}}$.

Noise can be accounted for in the stochastic Schrödinger equation in different but essentially equivalent ways. One possibility is to let the noise represent vacuum and/or thermal fluctuations of the environment; it is then natural to speak of input noise. Alternatively, we may let the noisy part of the output of the system appear as stochastic driving. Both forms of the noise are of course related to one another, as described by the input-output formalism of Collet and Gardiner 29. Depending on which form of the noise is chosen in the stochastic Schrödinger equation, the latter 
takes a linear or nonlinear appearance. The equivalence of the two formulations, recently shown by Ghirardi, Pearle and Rimini [15], by Belavkin and Staszewski [17] and by Goetsch and Graham [21,22], will be illustrated again below.

\section{LINEAR EVOLUTION FROM A CAT STATE}

We first discuss the solution of the linear stochastic Schrödinger equation 15

$$
d\left|\psi_{\theta}(t)\right\rangle=\left\{-\frac{\gamma}{2} a^{\dagger} a d t+\sqrt{\gamma} a e^{-i \varphi} d \theta(t)\right\}\left|\psi_{\theta}(t)\right\rangle
$$

Here $d \theta(t)$ is a real noise increment independent of its predecessors in time; otherwise, $d \theta(t)$ need not be specified at this point. The index $\theta$ on the wave vector is meant to indicate the contingency of that vector on the noise history: $\left|\psi_{\theta}(t)\right\rangle$ is a functional of the noise increments arisen in the past but independent of the one arising at present, $d \theta(t)$. To include the description of homodyning with a local oscillator of constant

phase $\varphi$ we have allowed for the phase factor $e^{-i \varphi}$ [12,22]. Obviously, the generator appearing on the rhs of the stochastic Schrödinger equation is non-Hermitian for which reason the normalization of the vector $\left|\psi_{\theta}(t)\right\rangle$ is not preserved in time.

Due to its linearity in the state vector $\left|\psi_{\theta}(t)\right\rangle$ and the bilinearity of the generator in $a$ and $a^{\dagger}$, the stochastic Schrödinger equation (2.1) can be solved rigorously. For instance, initial coherent states $| \pm \alpha\rangle$, defined as eigenstates of $a$ with eigenvalues $\pm \alpha$, evolve into 21,22

$$
c_{ \pm}(t)\left| \pm \alpha e^{-\frac{\gamma}{2} t}\right\rangle
$$

i. e. coherent states of amplitudes $\pm \alpha e^{-\frac{\gamma}{2} t}$ multiplied with stochastic factors of nonunit modulus,

$$
c_{ \pm}(t)=\exp \left\{\frac{1}{2}\left(\alpha^{2}+|\alpha|^{2}\right)\left(e^{-\gamma t}-1\right) \pm \alpha \sqrt{\gamma} \int_{0}^{t} e^{-\frac{\gamma}{2} s} d \theta(s)\right\}
$$


Of special interest to us will be an initial superposition of two coherent states,

$$
|\psi(t=0)\rangle=\frac{1}{\sqrt{2}}(|\alpha\rangle+|-\alpha\rangle) .
$$

For large values of the amplitudes, $|\alpha| \gg 1$, for which the constituents $| \pm \alpha\rangle$ become "macroscopically distinguishable", such a superposition is customarily called a Schrödinger cat state. Invoking the superposition principle we obtain the state originating from the superposition (2.4) as

$$
\left|\psi_{\theta}(t)\right\rangle=\frac{1}{\sqrt{2}}\left(c_{+}(t)\left|\alpha e^{-\frac{\gamma}{2} t}\right\rangle+c_{-}(t)\left|-\alpha e^{-\frac{\gamma}{2} t}\right\rangle\right) \text {. }
$$

We can now address the mean of the quadrature component

$$
X_{\varphi}=\frac{1}{2}\left(a e^{-i \varphi}+a^{\dagger} e^{i \varphi}\right)
$$

with respect to the normalized version of the time-evolved cat state (2.5). To avoid unnecessary complication we shall henceforth take the amplitude $\alpha$ as real and thus get

$$
\begin{aligned}
&\left\langle X_{\varphi}(t)\right\rangle_{\theta}= \mathcal{N}_{\theta}{ }^{-1}\left\langle\psi_{\theta}(t)\left|X_{\varphi}\right| \psi_{\theta}(t)\right\rangle \\
&= \mathcal{N}_{\theta}{ }^{-1}(\alpha / 2) e^{-\frac{\gamma}{2} t}\left\{\left(\left|c_{+}\right|^{2}-\left|c_{-}\right|^{2}\right) \cos \varphi\right. \\
&\left.\quad+i\left(c_{+}^{\star} c_{-}-c_{+} c_{-}^{\star}\right)\left\langle\alpha e^{-\frac{\gamma}{2} t} \mid-\alpha e^{-\frac{\gamma}{2} t}\right\rangle \sin \varphi\right\}, \\
& \mathcal{N}_{\theta}=\left\langle\psi_{\theta}(t) \mid \psi_{\theta}(t)\right\rangle .
\end{aligned}
$$

Clearly, this mean displacement vanishes initially, due to the symmetry of the initial state, and for large times, $t \gg 1 / \gamma$, due to the decay of $\left|\psi_{\theta}(t)\right\rangle$. However, at intermediate times we encounter a nonvanishing functional of the noise $\theta(t)$. The dependence of $\left\langle X_{\varphi}(t)\right\rangle_{\theta}$ on the phase of the local oscillator is interesting: For $\varphi=0$ the overlap of the two normalized coherent states $\left| \pm \alpha e^{-\frac{\gamma}{2} t}\right\rangle$,

$$
\left\langle\alpha e^{-\frac{\gamma}{2} t} \mid-\alpha e^{-\frac{\gamma}{2} t}\right\rangle=\exp \left(-2 \alpha^{2} e^{-\gamma t}\right)
$$


enters only through the normalization factor $\mathcal{N}_{\theta}$; for $\varphi=\pi / 2$, on the other hand, the presence of that overlap is essential for the transient appearance of a finite mean $\left\langle X_{\pi / 2}(t)\right\rangle_{\theta}$, the exponential smallness of $\langle\alpha \mid-\alpha\rangle=\exp \left(-2 \alpha^{2}\right)$ notwithstanding. Having pointed out this delicate dependence on the phase $\varphi$ we proceed to setting $\varphi=0$ and writing $X$ instead of $X_{0}$, for simplicity.

We shall mostly be concerned with times small compared to the "mechanical" relaxation time $1 / \gamma$. In that regime the mean displacement takes the simple form

$$
\langle X(t)\rangle_{\theta}=\alpha \tanh (2(\alpha \sqrt{\gamma}) \theta(t)), \quad t \ll 1 / \gamma
$$

this results by approximating as $e^{-\frac{\gamma}{2} t} \approx 1$ and dropping the exponentially small overlap $\langle\alpha \mid-\alpha\rangle$. We should note that the conditional mean $\langle X(t)\rangle_{\theta}$, being a functional of the noise $\theta(s)$ in $0 \leq s<t$, is a random quantity itself. It fluctuates from one realization of the process $\theta(t)$ to another one, each of which one might feel tempted to associate with a particular run of an experiment.

In order to compute full quantum averages of oscillator observables $Y$ like the displacement $X$ we still have to average over the noise $\theta(t)$ which we now need to specify. We want to describe an oscillator weakly interacting with a zero-temperature reservoir. By invoking the usual Born and Markov approximation, the stochastic Schrödinger equation was derived in [17,21,22], together with the prescription for calculating quantum means

$$
\langle Y(t)\rangle=\int d \mu_{t}^{W}(\{\theta\})\left\langle\psi_{\theta}(t)|Y| \psi_{\theta}(t)\right\rangle
$$

We here encounter the Wiener measure $d \mu_{t}^{W}(\{\theta\})$ for finding a realization $\theta(s)$ of the noise during the time interval $0 \leq s<t$. The result (2.10) allows us to think of $\theta(t)$ as the vacuum fluctuations of the reservoir forced on the oscillator as an "input".

An alternative useful way of thinking of the noise is suggested by introducing the normalized version of the state $\left|\psi_{\theta}(t)\right\rangle$ 


$$
\langle Y(t)\rangle_{\theta}=\left\langle\psi_{\theta}(t)|Y| \psi_{\theta}(t)\right\rangle /\left\langle\psi_{\theta}(t) \mid \psi_{\theta}(t)\right\rangle
$$

and rewriting (2.10) as

$$
\begin{aligned}
\langle Y(t)\rangle & =\int d \mu_{t}^{W}(\{\theta\})\left\langle\psi_{\theta}(t) \mid \psi_{\theta}(t)\right\rangle\langle Y(t)\rangle_{\theta} \\
& \equiv \int d \mu_{t}(\{\theta\})\langle Y(t)\rangle_{\theta}
\end{aligned}
$$

We may interpret

$$
d \mu_{t}(\{\theta\})=d \mu_{t}^{W}(\{\theta\})\left\langle\psi_{\theta}(t) \mid \psi_{\theta}(t)\right\rangle
$$

as a noise measure as well [15 22]; indeed, by choosing $Y=1$ in (2.12) we have the correct normalization $\int d \mu_{t}(\{\theta\})=1$. As was noted in [17,21,22 one may regard the random process with the measure $d \mu_{t}(\theta)$ as the output of the oscillator driven by vacuum fluctuations, i. e. a Wiener process $d \xi(t)$ as input,

$$
d \theta(t)=2 \sqrt{\gamma}\left\langle X_{\varphi}(t)\right\rangle_{\theta} d t+d \xi(t)
$$

Here, we have intentionally restored the index $\varphi$ on the quadrature component $X_{\varphi}$ since the input-ouput connection (2.14) remains in fact valid for arbitrary values of the phase of the local oscillator.

The two interpretations of the noise suggest different possibilities of calculating the means $\langle Y(t)\rangle$ by numerical simulation. One, and in fact the simpler one, is to generate random numbers with the Wiener measure $d \mu_{t}^{W}$, use them in evaluating $\left|\psi_{\theta}(t)\right\rangle$, and then employ (2.10). According to the second strategy one would again construct realizations of the Wiener process and a set of ensuing $\left|\psi_{\theta}(t)\right\rangle$ but then proceed to the new measure $d \mu_{t}$ and finally average as required in (2.12). We should stress that the expectation value $\langle X(t)\rangle_{\theta}$ in the definition (2.14) is meant in the sense of the second strategy, i. e. conditioned on the measure $d \mu_{t}$ for the past history $\theta(s)$, $s \leq t$. 
We now propose a closer inspection of the measure $d \mu_{t}(\theta)$ for times $0 \leq t \ll 1 / \gamma$. As already noted above we may

then drop the overlap $\left\langle\alpha e^{-\frac{\gamma}{2} t} \mid-\alpha e^{-\frac{\gamma}{2} t}\right\rangle \approx\langle\alpha \mid-\alpha\rangle$, thus obtaining

$$
\begin{aligned}
\left\langle\psi_{\theta}(t) \mid \psi_{\theta}(t)\right\rangle & =\frac{1}{2}\left(\left|c_{+}\right|^{2}+\left|c_{-}\right|^{2}\right) \\
& =e^{-2 \alpha^{2} \gamma t} \cosh \left[2 \alpha \sqrt{\gamma} \int_{0}^{t} d \theta(s)\right], \quad t \ll 1 / \gamma .
\end{aligned}
$$

In writing out the measure $d \mu_{t}(\{\theta\})$ more explicitly it is convenient to discretize the time as $t_{n}=n \Delta t, n=0 \ldots N, t_{N}=t$ and to consider increments $\Delta_{n} \theta$ such that $\theta\left(t_{n}\right)=\sum_{i=0}^{n-1} \Delta_{i} \theta$. Assuming $\theta(0)=0$ we then have

$$
\begin{aligned}
d \mu_{t}(\{\theta\})=\frac{1}{2}[ & \prod_{i=0}^{N-1} \frac{d \Delta_{i} \theta}{\sqrt{2 \pi \Delta t}} \exp \left(-\left(\Delta_{i} \theta-2 \alpha \sqrt{\gamma} \Delta t\right)^{2} / 2 \Delta t\right) \\
& \left.+\prod_{i=0}^{N-1} \frac{d \Delta_{i} \theta}{\sqrt{2 \pi \Delta t}} \exp \left(-\left(\Delta_{i} \theta+2 \alpha \sqrt{\gamma} \Delta t\right)^{2} / 2 \Delta t\right)\right] .
\end{aligned}
$$

When averaging the mean displacement (2.9) with this measure we may first reduce $d \mu_{t}(\{\theta\})$ to the marginal density of the single variable $\theta(t) \equiv \theta$

$$
\begin{aligned}
P(\theta, t) & =\int d \mu_{t}(\{\theta\}) \delta\left(\theta-\sum_{i=0}^{N-1} \Delta_{i} \theta\right) \\
& =\frac{1}{2}\left\{P^{(+)}(\theta, t)+P^{(-)}(\theta, t)\right\} \\
P^{( \pm)} & =\frac{1}{\sqrt{2 \pi t}} \exp \left\{-(\theta \mp 2(\alpha \sqrt{\gamma}) t)^{2} / 2 t\right\}
\end{aligned}
$$

The two Gaussians here appearing are not resolved from one another as long as their separation $4(\alpha \sqrt{\gamma}) t$ is smaller than their width $\sqrt{t}$. However, as soon as

$$
t \gg \frac{1}{2 \gamma \alpha^{2}} \equiv t_{\mathrm{dec}}
$$

the density $P(\theta, t)$ has two non-overlapping peaks. Since the so-called decoherence time $1 / 2 \gamma \alpha^{2}$ [27,28] is, for strongly excited coherent states $\left(\alpha^{2} \gg 1\right)$, much shorter than the mechanical life time of excitations $1 / \gamma$, the separation in question takes place well within the range of validity $(t \ll 1 / \gamma)$ of the various simplifications made 
in arriving at (2.17). We may conclude that the displacement of the oscillator has an overwhelming likelihood, at all times in the interval $1 / 2 \gamma \alpha^{2} \ll t \ll 1 / \gamma$, to take on either one or the other of two "macroscopically" distinguishable values rather than their vanishing ensemble average. Fig. 1 shows the density $P(\theta, t)$ for various moments of time.

Moreover, one intuitively expects jumps between the two macroscopically distinct values of $\langle X(t)\rangle_{\theta}$ to become less and less frequent as $t$ begins to exceed the decoherence time $t_{\mathrm{dec}}$. In order to substantiate this expectation we consider the joint density

$$
P\left(\theta, t ; \theta^{\prime}, t^{\prime}\right)=\int d \mu_{t^{\prime}}(\{\theta\}) \delta\left(\theta-\sum_{i=1}^{N-1} \Delta_{i} \theta\right) \delta\left(\theta^{\prime}-\sum_{j=1}^{N^{\prime}-1} \Delta_{j} \theta\right)
$$

with $t^{\prime}=N^{\prime} \Delta t \geq t=N \Delta t$. By noting $\theta^{\prime}-\theta=\sum_{i=N}^{N^{\prime}-1} \Delta_{i} \theta$ and doing the Gaussian integrals we arrive at

$$
\begin{aligned}
& P\left(\theta, t ; \theta^{\prime}, t^{\prime}\right)=\frac{1}{2}\left\{P^{(+)}(\theta, t) P^{(+)}\left(\theta^{\prime}-\theta, t^{\prime}-t\right)\right. \\
& \left.+P^{(-)}(\theta, t) P^{(-)}\left(\theta^{\prime}-\theta, t^{\prime}-t\right)\right\} .
\end{aligned}
$$

It is interesting to realize how the double-peak structure (2.17) of the singlepoint density generalizes to the two-point density (2.20). In particular, the absence of cross terms $P^{(+)} P^{(-)}$suggests that there is no interference between what happens near $\langle X\rangle_{\theta} \approx+\alpha$ and near $\langle X\rangle_{\theta} \approx-\alpha$. For a quantitative estimate of the frequency of jumps between the two branches we measure $t$ and $t^{\prime}$ in units of the decoherence time $\left(t=\tau / 2 \gamma \alpha^{2}\right)$ and evaluate the probability of finding $\theta$ within a width $\sqrt{t}$ around the peak at $+2(\alpha \sqrt{\gamma}) t$, and $\theta^{\prime}$ within a width $\sqrt{t^{\prime}}$ around the peak at $-2(\alpha \sqrt{\gamma}) t^{\prime}$,

$$
\begin{array}{r}
p\left(\tau, \tau^{\prime}\right)=\operatorname{prob}\left\{\theta\left(\frac{\tau}{2 \gamma \alpha^{2}}\right) \in\left[\frac{1}{\alpha \sqrt{\gamma}}(\tau-\sqrt{\tau / 8}), \frac{1}{\alpha \sqrt{\gamma}}(\tau+\sqrt{\tau / 8})\right]\right. \\
\text { and } \left.\theta^{\prime}\left(\frac{\tau^{\prime}}{2 \gamma \alpha^{2}}\right) \in\left[\frac{1}{\alpha \sqrt{\gamma}}\left(\tau^{\prime}-\sqrt{\tau^{\prime} / 8}\right), \frac{1}{\alpha \sqrt{\gamma}}\left(\tau^{\prime}+\sqrt{\tau^{\prime} / 8}\right)\right]\right\}
\end{array}
$$


We immediately obtain this "jump probability" as

$$
p\left(\tau, \tau^{\prime}\right)=\frac{1}{2 \pi}\left\{\int_{\mathrm{I}_{1}} d x e^{-x^{2}} \int_{\mathrm{I}_{2}} d y e^{-y^{2}}+\int_{\mathrm{I}_{1}^{\prime}} d x e^{-x^{2}} \int_{\mathrm{I}_{2}^{\prime}} d y e^{-y^{2}}\right\}
$$

with the integration intervals

$$
\begin{aligned}
& \mathrm{I}_{1}=[-1 / \sqrt{8},+1 / \sqrt{8}] \\
& \mathrm{I}_{2}=\frac{1}{\sqrt{\tau-\tau^{\prime}}}\left[-2 \tau^{\prime}-\tau x-\sqrt{\tau^{\prime} / 8},-2 \tau^{\prime}-\tau x+\sqrt{\tau^{\prime} / 8}\right] \\
& \mathrm{I}_{1}^{\prime}=[2 \sqrt{\tau}-1 / \sqrt{8}, 2 \sqrt{\tau}+1 / \sqrt{8}] \\
& \mathrm{I}_{2}^{\prime}=\frac{1}{\sqrt{\tau^{\prime}-\tau}}\left[-\tau x-\sqrt{\tau^{\prime} / 8},-\tau x+\sqrt{\tau^{\prime} / 8}\right] .
\end{aligned}
$$

Now first consider $\tau \approx 1, \tau^{\prime} \gg 1$. Then the first summand in the jump probability (2.22) becomes negligibly small since $\mathrm{I}_{2}$ covers a relatively small interval far out in the wing of the Gaussian integrand; the second summand in (2.22), however, approaches a limit independent of $\tau^{\prime}$ since $\mathrm{I}_{2}^{\prime} \rightarrow[-1 / \sqrt{8},+1 / \sqrt{8}]$; therefore, the jump probability $p\left(\tau \approx 1, \tau^{\prime} \gg 1\right)$ is not small compared to unity. However, now consider what happens for large times, $\tau^{\prime}>\tau \gg 1$; then both summands in (2.22) tend to become vanishingly small, i. e. of the order $e^{-4 \tau^{2}}$ as $\tau$ grows large. Indeed, once one has waited several decoherence times after the preparation of the cat state, jumps between the macroscopically distinguishable values $\langle X\rangle_{\theta}= \pm \alpha$ become exceedingly unlikely. This behavior is fully borne out by numerical simulations, as exemplified in Fig. 2 where two "trajectories" $\langle X(t)\rangle_{\theta}$ pertaining to two realizations of the noise $\theta(t)$ are displayed.

The foregoing reasoning makes for a certain temptation to associate single realizations of the noise $\theta(t)$ and the accompanying $\langle X(t)\rangle_{\theta}$ with single runs of an experiment. We shall discuss the legitimacy of such an interpretation further below. It may be appropriate, however, to right away ease the temptation mentioned by throwing a glance at Fig. 3 which depicts a simulation of $\left\langle X_{\pi / 2}(t)\right\rangle_{\theta}$ for a single realization of the noise $\theta(t)$. Obviously, in contrast to $\left\langle X_{0}(t)\right\rangle_{\theta}$ the mean of the 
quadrature component $X_{\pi / 2}$ shows no tendency towards choosing one of the two distinguished values $\pm \alpha \exp \left(-\frac{\gamma}{2} t\right)$; even until times of the order of the mechanical relaxation time $1 / \gamma$ the mean $\left\langle X_{\pi / 2}(t)\right\rangle_{\theta}$ stays close to zero before beginning to display some sizable fluctuations and then eventually quieting down at zero again for $t \gg 1 / \gamma$. This behavior [30, 31] of $\left\langle X_{\pi / 2}(t)\right\rangle_{\theta}$ is easily understood by recalling the remark on the dependence of that mean on the phase $\varphi$ of the local oscillator, made after (2.7); for $\varphi=\pi / 2$ one must wait until the overlap (2.8) of the two coherent states $\left| \pm \alpha \exp \left(-\frac{\gamma}{2} t\right)\right\rangle$ has grown to values of order unity before one can expect to see sizable fluctuations of the mean $\left\langle X_{\pi / 2}(t)\right\rangle_{\theta}$ away from zero.

\section{EVOLUTION OF CAT STATES ACCORDING TO THE NONLINEAR SCHRÖDINGER EQUATION}

In 15, 17,21,22 the linear stochastic Schrödinger equation (2.1) was shown to be equivalent to the following nonlinear stochastic Schrödinger equation

$$
\begin{aligned}
d\left|\phi_{\xi}(t)\right\rangle=\{- & \frac{\gamma}{2}\left(a^{\dagger} a-2 a\langle X(t)\rangle_{\xi}+\langle X(t)\rangle_{\xi}^{2}\right) d t \\
& \left.+\sqrt{\gamma}\left(a-\langle X(t)\rangle_{\xi}\right) d \xi(t)\right\}\left|\phi_{\xi}(t)\right\rangle
\end{aligned}
$$

where the mean displacement $\langle X(t)\rangle_{\xi}=\frac{1}{2}\left\langle a+a^{\dagger}\right\rangle_{\xi}$ is meant with respect to the state $\left|\phi_{\xi}\right\rangle$ itself,

$$
\langle X(t)\rangle_{\xi}=\left\langle\phi_{\xi}(t)\left|\frac{1}{2}\left(a+a^{\dagger}\right)\right| \phi_{\xi}(t)\right\rangle
$$

The noise increment $d \xi(t)$ represents a Wiener process. The derivation of the nonlinear evolution equation (3.1) from the linear one, (2.1) proceeds in two steps 15, 17,21,22]. First, one interprets the noise $d \theta(t)$ in (2.1) as output noise driven by a Wiener input $d \xi(t)$ according to (2.14). By then normalizing as

$$
\left|\phi_{\xi}\right\rangle=\left|\psi_{\theta(\{\xi\})}\right\rangle / \sqrt{\left\langle\psi_{\theta(\{\xi\})} \mid \psi_{\theta(\{\xi\})}\right\rangle}
$$


one arrives at (3.1). This equation has been employed previously by several authors (see e. g. [12 [14).

Due to the equivalence of the linear and the nonlinear stochastic Schrödinger equation and due to the existence of a rigorous solution of the linear equation, it should also be possible to construct the exact solution of the nonlinear equation. This has in fact been achieved recently by Carmichael, Kochan and Tian [30] in the following way. One removes part of the nonlinearity from the nonlinear stochastic Schrödinger equation (3.1) by working with the non-normalized wave vector $\left|\psi_{\theta(\{\xi\})}(t)\right\rangle$ (see (3.3)), thus obtaining

$$
d\left|\psi_{\theta(\{\xi\})}(t)\right\rangle=\left\{-\frac{\gamma}{2}\left(a^{\dagger} a-2 a\langle X(t)\rangle_{\xi} d t+\sqrt{\gamma} a d \xi(t)\right\}\left|\psi_{\theta(\{\xi\})}(t)\right\rangle .\right.
$$

The ansatz

$$
\left|\psi_{\theta(\{\xi\})}(t)\right\rangle=e^{\alpha^{2}\left(e^{-\gamma t}-1\right)}\left(e^{\chi(t)}\left|\alpha e^{-\frac{\gamma}{2} t}\right\rangle+e^{-\chi(t)}\left|-\alpha e^{-\frac{\gamma}{2} t}\right\rangle\right)
$$

yields an Ito differential equation for the random process $\chi(t)$. The associated FokkerPlanck equation for the density $P(\chi, t)$ happens to allow for the solution given in [30]. After simplifying as before, i. e. $e^{-\gamma t}-1 \rightarrow-\gamma t,\langle\alpha \mid-\alpha\rangle \approx 0$, the solution $P(\chi, t)$ originating from $P(\chi, 0)=\delta(\chi)$ takes the form of a sum of two Gaussians,

$$
P(\chi, t)=\frac{1}{2 \sqrt{2 \pi \alpha^{2} \gamma t}}\left(e^{\left(\chi+2 \alpha^{2} \gamma t\right)^{2} / 2 \alpha^{2} \gamma t}+e^{\left(\chi-2 \alpha^{2} \gamma t\right)^{2} / 2 \alpha^{2} \gamma t}\right)
$$

which in structure resembles our density (2.17) of the output noise $\theta(t)$. It is most interesting to again see the width of each of the two Gaussians grow as $\sqrt{t}$ while their separation grows as $t$, i. e. much faster. Moreover, we again find the separation to become manifest after a time of the order of the decoherence time $t_{\mathrm{dec}}=1 / 2 \gamma \alpha^{2}$.

The equivalence of the two versions (2.1) and (3.1) of the stochastic Schrödinger equation is worth one more illustration. The nonlinear equation (3.1) is easily seen to imply the following equation for the mean $\langle X(t)\rangle_{\xi}$ defined in (3.2), 


$$
d\langle X(t)\rangle_{\xi}=2 \sqrt{\gamma}\left(\alpha^{2}-\langle X(t)\rangle_{\xi}^{2}\right) d \xi(t)
$$

The Ito integral of this equation takes the form

$$
\langle X(t)\rangle_{\xi}=\alpha \tanh \left(2(\alpha \sqrt{\gamma}) \xi(t)+4 \gamma \alpha \int_{0}^{t} d t^{\prime}\left\langle X\left(t^{\prime}\right)\right\rangle_{\xi}\right)
$$

Obviously, we have arrived at an integral equation rather than an explicit solution. However, the equivalence of the integral equation (3.8) with the solution (2.9) of the linear Schrödinger equation can clearly be seen with the help of eq. (2.14).

\section{PREPARATION OF A CAT STATE}

Imagine a spin $1 / 2$ (or equivalently, a two-level atom) prepared in an eigenstate of the component $S_{x}$ with eigenvalue $+\hbar / 2$. In the $S_{z}$ representation that state will take the form

$$
\left.\left.\left.\left.\frac{1}{\sqrt{2}}(\mid+)+\mid-\right)\right) \text { with } S_{z} \mid \pm\right)= \pm \frac{\hbar}{2} \mid \pm\right) \text {. Let, on the other hand, an harmonic }
$$

oscillator be prepared in the vacuum state $|0\rangle$, with $a|0\rangle=0$. If we couple the two systems impulsively according to the Hamiltonian

$$
H(t)=\delta(t) i 2\left(\alpha a^{\dagger}-\alpha^{*} a\right) S_{z}
$$

we will produce the composite state

$$
\left.\left.\frac{1}{\sqrt{2}}(|\alpha\rangle \mid+)+|-\alpha\rangle \mid-\right)\right)
$$

in which the coherent state $|\alpha\rangle$ of the oscillator is correlated with the "up" state $\mid+$ ) of the spin while the coherent state of the opposite amplitude, $|-\alpha\rangle$, is correlated with the spin-down-state $\mid-$ ). Needless to say, the composite state (4.2) is not a cat state of the oscillator, even if the amplitude $\alpha$ is large.

In order to proceed towards preparing a cat state we let the spin be exposed to a magnetic field in the $y$ direction such that spin states are transformed as 


$$
\left.\left.\left.\mid \pm) \rightarrow e^{-i \pi S_{y} / 2 \hbar} \mid \pm\right)=\frac{1}{\sqrt{2}}(\mid+) \pm \mid-\right)\right)
$$

This corresponds to a spin rotation by $\pi / 2$ about the $y$ axis. The composite state (4.2) is thus transformed into

$$
\left.\left.\frac{1}{\sqrt{2}}\{\mid+) \frac{1}{\sqrt{2}}(|\alpha\rangle+|-\alpha\rangle)+\mid-\right) \frac{1}{\sqrt{2}}(|\alpha\rangle-|-\alpha\rangle)\right\} .
$$

Now both eigenstates of $S_{z}$ are correlated with cat states of the oscillator, if $|\alpha| \gg 1$.

We may finally imagine a measurement of $S_{z}$. Every time we find $S_{z}=+\hbar / 2$ we know that the oscillator is prepared in the cat state

$$
\frac{1}{2}(|\alpha\rangle+|-\alpha\rangle) .
$$

In principle, the thought experiment just sketched can be realized by playing with two level atoms traversing microwave or optical cavities. Care would have to be taken that the two-step preparation takes less time than a decoherence time $t_{\mathrm{dec}}=1 / 2 \gamma|\alpha|^{2}$ where $\gamma$ is the damping constant of the cavity within which the cat state is to be produced.

\section{CONCLUSION AND DISCUSSION}

Mostly employing a linear version of the stochastic Schrödinger equation we have presented a rigorous description of the fate of an initial state of the cat type for the damped harmonic oscillator. While our description is stochastically equivalent to one using the master equation for the density operator, it has the additional appeal of being more closely related to individual runs of an experiment rather than to ensembles of such. Indeed, the fate of the cat state described here makes for a certain temptation to associate a single realization of the noise $\theta(t)$ and the accompanying mean displacement $\langle X(t)\rangle_{\theta}$ with a single run of an experiment. In fact, such an interpretation is known to be legitimate only with respect to balanced homodyne 
experiments where the output of the quantum oscillator is put to interference with a local oscillator of large amplitude and stable phase $\varphi=0$; sharp values are then in effect recorded for the output noise $\theta(t)$ of the quantum oscillator.

Inasmuch as the mean displacement $\langle X(t)\rangle_{\theta}$ tends to assume, for times in the interval $1 / \gamma \alpha^{2} \ll t \ll 1 / \gamma$, either one of the two values $\pm \alpha$, one may even feel inclined towards regarding the displacement $X$ itself as a measured observable. The exceedingly small likelihood of jumps between the two preferred values $\pm \alpha$ during the time interval mentioned does permit some such indulgence. One must keep in mind, though, that both for early times, $t \approx 1 / \gamma \alpha^{2}$, and large times, $t \approx 1 / \gamma$, the displacement cannot be considered as sharp in an individual run of the experiment; nor can the quadrature component $X_{\pi / 2}(t)$ at any time.

Some further caution is indicated against hurried conclusions for quantum measurement theory. The heat bath providing the damping of the quantum oscillator is, strictly speaking, not the one needed for "objectivation" of sharp "pointer readings"; nor is the displacement $X$ really playing the role of a pointer variable. In our context the quantum oscillator rather acts as a measured object and objectivation only arises in the photodetector which generates a macroscopic electric current. The pointer variable with respect to which the detector must secure decoherence, i. e. objectivation, is just the output noise $\theta(t)$ for which sharp values are recorded. A striking manifestation of the potential "nonobjectivity" of the interaction of the oscillator with the heat bath may also be seen in the sensitive dependence of the statistics of $X_{\varphi}(t)$ on the phase $\varphi$ of the local oscillator, discussed in Section 【1 and already noted in 30,31. Astounding as that sensitivity may be, it does not make for worries in the context of quantum measurement theory; while the phase of the local oscillator does not influence the interaction of the quantum oscillator with its damping reservoir, that phase does enter the oscillator observable coupled to the detector, 
i. e. the ouput noise $\theta(t)$. The interested reader is referred to Zurek's discussion of "pointer bases" with respect to which fast decoherence takes place [25].

\section{ACKNOWLEDGMENTS}

We gratefully acknowledge support by the Sonderforschungsbereich "Unordnung und grosse Fluktuationen" of the Deutsche Forschungsgemeinschaft. We thank Howard Carmichael for sending us preprints of his work prior to publication. 


\section{REFERENCES}

[1] P. Pearle, Phys. Rev. D 13, 857 (1976).

[2] N. Gisin, Phys. Rev. Lett. 52, 1657 (1984).

[3] L. Diósi, Phys. Lett. A 129, 419 (1988).

[4] J. Dalibard, C. Cohen-Tannoudji, J. Opt. Soc. Am. B 6, 2023 (1989).

[5] N. Gisin, Helv. Phys. Acta 62, 363 (1989).

[6] N. Gisin, I. C. Percival, J. Phys. A 25, 5677 (1992); Phys. Lett. A 167, 315 (1992).

[7] J. Dalibard, Y. Castin, K. Mølmer, Phys. Rev. Lett. 68, 580 (1992).

[8] R. Dum, P. Zoller, H. Ritsch, Phys. Rev. A 45, 4879 (1992).

[9] C. W. Gardiner, A. S. Parkins, P. Zoller, Phys. Rev. A 46, 4363 (1992).

[10] R. Dum, A. S. Parkins, P. Zoller, C. W. Gardiner, Phys. Rev. A 46, 4382 (1992).

[11] K. Mølmer, Y. Castin, J. Dalibard, J. Opt. Soc. Am. B 10, 524 (1993).

[12] H. M. Wiseman, G. J. Milburn, Phys. Rev. A 47, 642 (1993); Phys. Rev. A 47, 1652 (1993).

[13] H. J. Carmichael, An Open Systems Approach to Quantum Optics, Lecture Notes in Physics m18, Berlin, Springer 1993.

[14] P. Goetsch, R. Graham, Ann. Physik 2, 706 (1993).

[15] G. C. Ghirardi, P. Pearle, A. Rimini, Phys. Rev. A 42, 78 (1990).

[16] V. P. Belavkin, Phys. Lett. A 140, 355 (1989); J. Phys. A 31, L1109 (1989); J. Math. Phys. 31, 2930 (1990). 
[17] V. P. Belavkin, P. Staszewski, Phys. Rev. A 45, 1347 (1992).

[18] A. Barchielli, V. P. Belavkin, J. Phys. A 24, 1495 (1991).

[19] A. S. Holevo, in Quantum Aspects of Optical Communications, ed. by C. Bendjaballah, O. Hirota,

S. Reynaud, Lecture Notes in Physics vol. 376, Berlin, Springer 1991, p. 127.

[20] A. Barchielli, Int. J. Theor. Phys. 32, 2221 (1993).

[21] P. Goetsch, R. Graham, in Quantum Optics VI, ed. by J. D. Harvey, D. F. Walls, Berlin, Springer 1994.

[22] P. Goetsch, R. Graham, submitted to Phys. Rev. A.

[23] F. Haake, M. Zukowski, Phys. Rev. A 47, 2506 (1994).

[24] F. Haake, D. F. Walls, Phys. Rev. A 36, 730 (1987).

[25] W. H. Zurek, Phys. Today 44, 36 (1991), and references therein.

[26] B. M. Garraway, P. L. Knight, in Quantum Optics VI, ed. by J. D. Harvey, D. F. Walls, Berlin, Springer 1994;

B. M. Garraway, P. L. Knight, submitted to Phys. Rev. Lett.

[27] D. F. Walls, G. J. Milburn, Phys. Rev. A 31, 2403, 1985.

[28] C. W. Gardiner, Quantum Noise, Berlin, Springer 1991.

[29] M. J. Collet, C. W. Gardiner, Phys. Rev. A 30, 1386 (1984);

C. W. Gardiner, M. J. Collet, Phys. Rev. A 31, 3761 (1985).

[30] H. J. Carmichael, P. Kochan, L. Tian, in Proceedings of the International Symposium on Coherent States: Past, Present, and Future, ed. by J. R. Klauder, World Scientific, Singapore, in press. 
[31] H. J. Carmichael, in Quantum Optics VI, ed. by J. D. Harvey, D. F. Walls, Berlin, Springer 1994. 


\section{FIGURES}

FIG. 1. Marginal density of the output noise $\theta$ for different times $\tau$ measured in units of the decoherence time for parameter values $\gamma=0.1,|\alpha|=3$.

FIG. 2. The mean $\left\langle X_{0}(t)\right\rangle_{\theta}$ for two runs of the nonlinear stochastic Schrödinger equation with initial amplitudes $\alpha= \pm 3$. The dashed lines show the mechanical relaxation $\pm \alpha \exp (-\gamma t / 2)$ of the amplitudes of the coherent states. In (a), the approach to $+\alpha \exp (-\gamma t / 2)$ at $t \approx 0.75 t_{\mathrm{dec}}$ is not quite close enough to produce definite locking which occurs only at $t \approx 2.6 t_{\mathrm{dec}}$.

FIG. 3. The mean $\left\langle X_{\pi / 2}(t)\right\rangle_{\theta}$. Again, $\alpha= \pm 3$. 
This figure "fig1-1.png" is available in "png" format from: http://arxiv.org/ps/gr-qc/9407001v1 

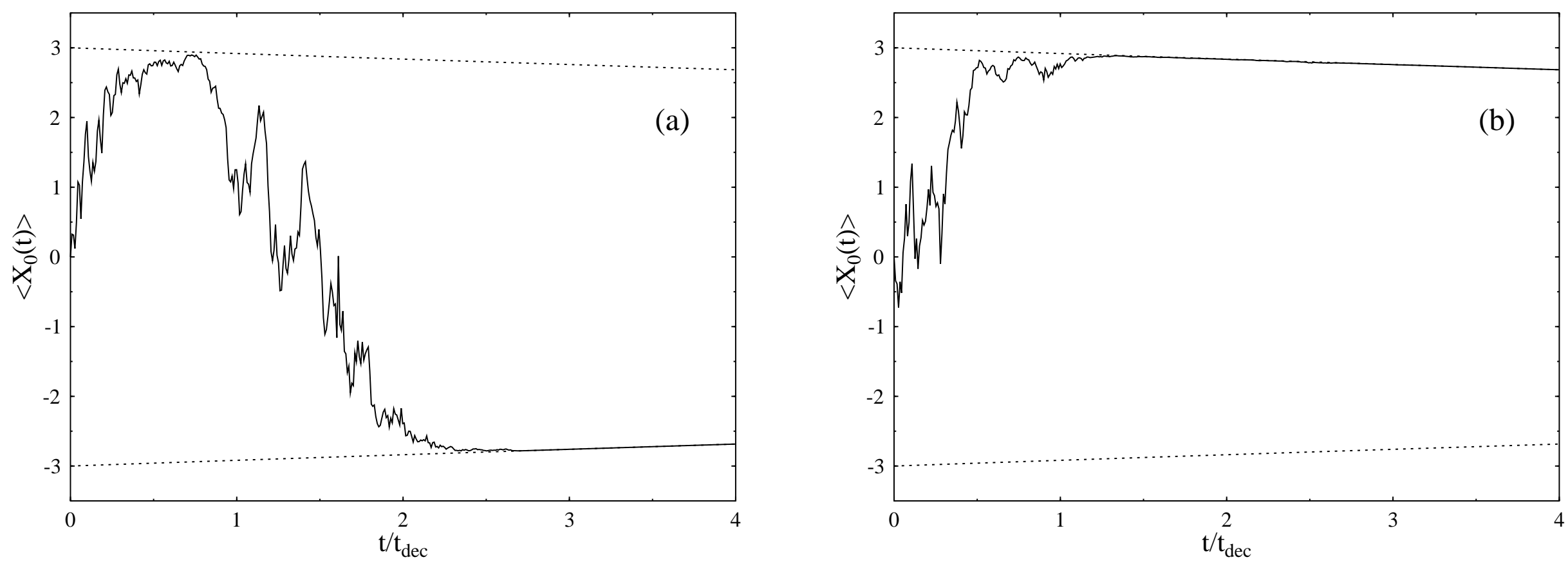
This figure "fig1-2.png" is available in "png" format from: http://arxiv.org/ps/gr-qc/9407001v1 


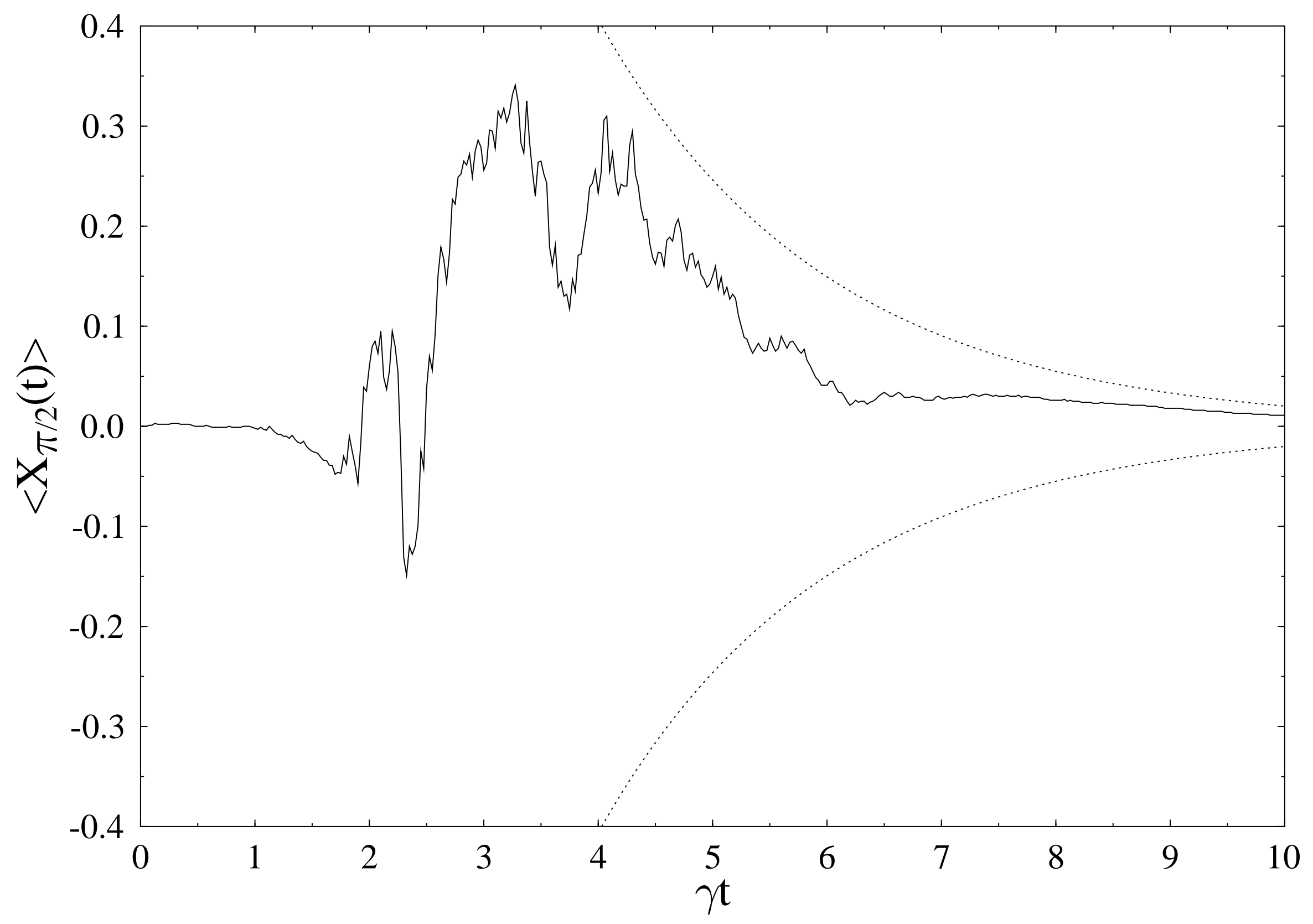


This figure "fig1-3.png" is available in "png" format from: http://arxiv.org/ps/gr-qc/9407001v1 


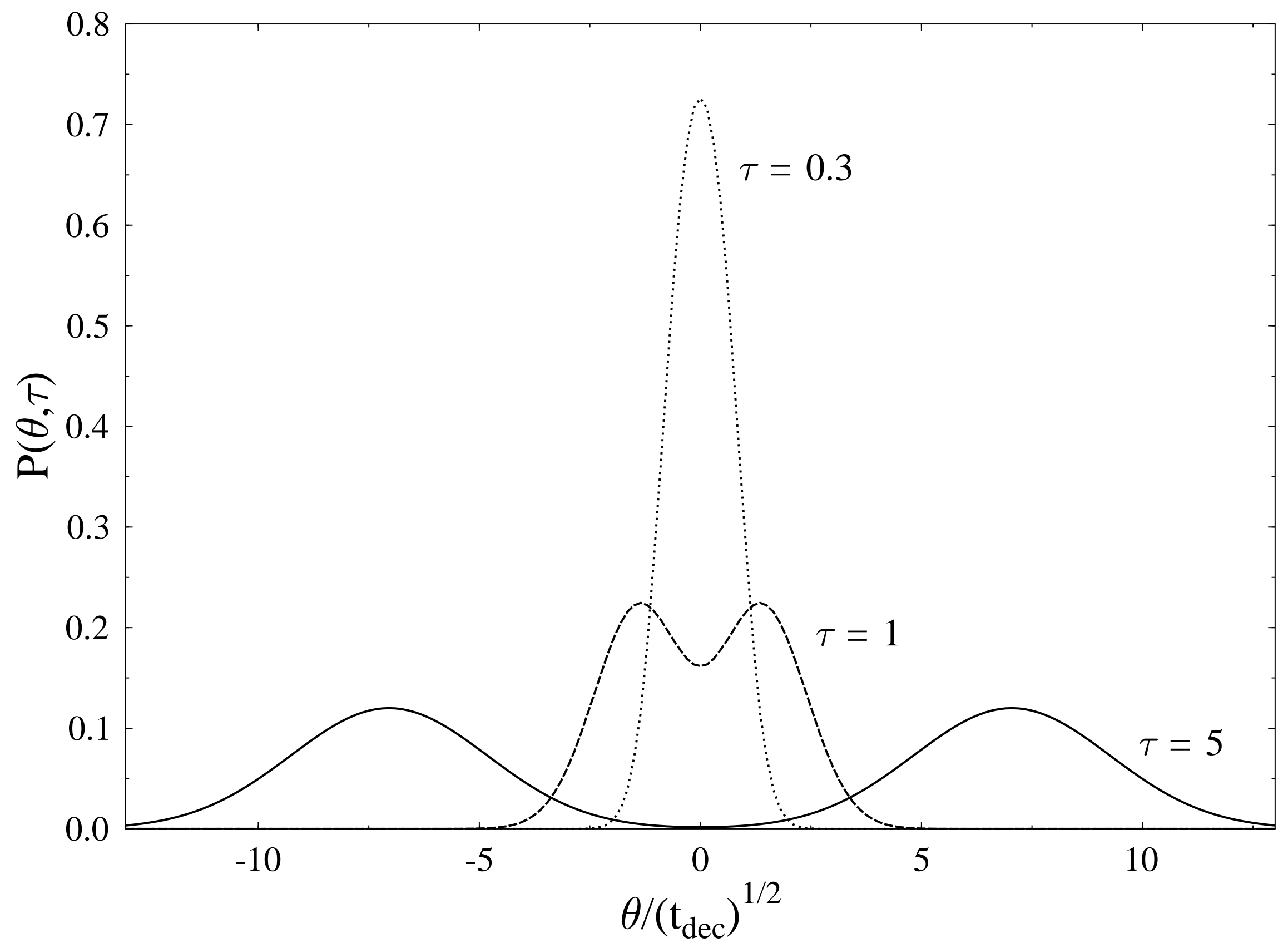

